Original Article

\title{
Quantitative postural analysis and pain in children and adolescents victims of burns
}

\author{
Paola Janeiro Valenciano, MSc ${ }^{1)^{*}}$, Edna Yukimi Itakussu, MSc ${ }^{1)}$, \\ Celita Salmaso Trelha, PhD²), Dirce Shizuko Fujisawa, PhD $^{2}$ ) \\ 1) Master's Program in Rehabilitation Sciences, Universidade Estadual de Londrina, Hospital \\ Universitário: Centro de Ciências da Saúde, Rua Robert Kock, 60 Vila Operária, 86038-350, \\ Londrina, PR, Brazil \\ 2) Department of Physical Therapy, Universidade Estadual de Londrina (UEL), Brazil
}

\begin{abstract}
Purpose] This study aimed to quantitatively assess postural alignment in both frontal and sagittal planes, as well as pain in children and adolescents victims of burn injuries. [Subjects and Methods] This crosssectional study included 21 victims of burns, nine children (age [mean $\pm \mathrm{SD}$ ], $7.3 \pm 1.1 \mathrm{yrs}$ ) and 12 adolescents $(12,0$ $\pm 1.4 \mathrm{yrs}$ ), classified as medium and large burns, being investigated on pain and postural alignment evaluated by photogrammetry. Pain intensity was assessed by face scales and postural examination included the assessment of global and thoraco-lumbo-pelvic alignment by previously designed protocols. [Results] Only two adolescents reported mild pain associated with burn injuries, whereas deviations of the projection of the gravity center; forward head posture, and scapular asymmetry were observed in both groups. In the analysis of the thoraco-lumbo-pelvic alignment, children tended to have anterior inclination trunk, increased thoracic kyphosis and lumbar lordosis, while in adolescents, increased thoracic kyphosis and lumbar lordosis were observed. [Conclusion] The results indicate that due to the postural alterations and asymmetries in both frontal and sagittal planes, there is an increased risk of developing scoliosis and possible future pain. Thus, physiotherapy is indicated and should be maintained until complete growth is reached.

Key words: Burns, Physical Therapy Specialty, Posture
\end{abstract}

(This article was submitted Jul. 8, 2015, and was accepted Sep. 1, 2015)

\section{INTRODUCTION}

Burns are considered a major health problem especially in low and middle income countries where more than $95 \%$ of deaths caused by burns occur ${ }^{1}$. Children and adolescents are frequently affected by burns and physical and psychological consequences are more intense by reason of growth and development ${ }^{2}$. Furthermore, possible sequelae may reduce self-esteem and bring functional limitations in the long term ${ }^{3}$. Most accidents are caused by contact with fire, overheated liquids and surfaces, smoke inhalation, chemical, electrical or radioactive burns ${ }^{4)}$. First-degree burns may be managed in emergency rooms, while second-degree ones require a specialist evaluation for possible excision and grafting ${ }^{5}$. According to Berman et al. ${ }^{6}$, the healing phase may last up to two years, and this is a critical period in which keloids and hypertrophic scars may form, as well as chronic pain associated to burn injuries ${ }^{7)}$. Burn complications include musculoskeletal consequences that may occur in the

*Correspondending author. Paola Janeiro Valenciano (E-mail: paola_jv3@hotmail.com)

C 2015 The Society of Physical Therapy Science. Published by IPEC Inc. This is an open-access article distributed under the terms of the Creative Commons Attribution Non-Commercial No Derivatives (by-ncnd) License $<$ http://creativecommons.org/licenses/by-nc-nd/3.0/> . first weeks or take years after the injury, affecting life quality substantially ${ }^{8}$.

Thus, due to broad implications of burns it is crucial that the entire medical team involved in the treatment use valid, sensitive and reliable measurement tools, in order to optimize the results, clinical management, and the performance of clinical research ${ }^{9,10)}$. Physiotherapy is important throughout the entire rehabilitation process, as it helps patients to reach optimal functional results whilst maining adequate posture, which is an essential part of the therapeutic goals ${ }^{11)}$. Postural evaluation by placing markers in specific anatomical regions, and later photographic analysis has been widely used to quantitatively assess different populations ${ }^{12,13,20)}$. Concerning victims of burns, postural complications have been sub-reported in literature and there is a lack of studies that evaluate those complications objectively. Therefore, the goal of this study is to quantitatively evaluate the pain and the postural alignment of children and adolescents victims of burn injuries in order to provide resources to prevent further postural alterations and possible sequelae, giving support to long-term care.

\section{SUBJECTS AND METHODS}

This is a cross-sectional study carried out in children and adolescents victims of burns, in the period of six months to two years after hospital discharge. All patients that returned 
to the ambulatory care unit of the Burn Injuries Treatment Center (BTC), during the period of data collection were evaluated. The study has been approved by the Ethics and Research Committee involving human beings of the institution. All participants and their parents gave written informed consent before the study.

The study was carried out from October 2013 to July 2014. It included children and adolescents victims of burn injuries according to the programmed outpatient return. The inclusion criteria were: the victims should be between six to 14 years old, with burn injuries diagnosis, had been discharged from hospital for a period of time between six months to two years. The exclusion criteria were: presence of sensorial deficiency; inability to stand without external assistance; chronical or acute musculoskeletal diseases; previous sequelae; unhealed wounds.

The data regarding the hospitalization period was collected through a study of patient records, and information regarding the outpatient physical care was obtained through interviews with the participants and their parents or legal guardians at the time of collection. To investigate pain intensity, face scales developed by Claro ${ }^{14)}$, were applied-a simple tool focused on the children population. The participants reported pain when choosing among five facial expressions available - being zero the value equivalent to pain absence, one referring to mild pain, two for moderate pain, three for severe pain and finally four for unbearable pain ${ }^{14)}$. Moreover, patients were asked to report the exact location of pain, and if it was related to the burn.

Evaluations were performed at the BTC ambulatory room, and patients were prepared by one physiotherapist trained to mark anatomical points, through location tutorial, elaborated by Ferreira ${ }^{15}$ ) for the standardization of photograph; furthermore they were asked to remain barefoot wearing either underwear or shorts and a top. Posture was assessed using a 16.1 Megapixels Nikon ${ }^{\circledR}$ digital camera (model COOLPIX P510), aluminum tripod-Tron ${ }^{\circledR}$ (model vpt-10). To obtain standardized images, ground markings were done so that the camera could be adequately positioned on the tripod at the height of $90 \mathrm{~cm}$ from the ground and at a distance of $3 \mathrm{~m}$ between the center of the camera and the wall. A non-reflective black cloth was placed to the wall and a $30 \times 35 \mathrm{~cm}$ ethyl vinyl acetate mat was nailed on the floor at a distance of $15 \mathrm{~cm}$ from the wall.

To collect the images, participants were instructed to remain in the standing position on the mat, in their usual and comfortable posture, with feet slightly apart, weight evenly distributed, and a straightforward look ${ }^{16)}$. The materials used were: $15 \mathrm{~mm}$ white styrofoam balls for the marking of anatomical points; $8 \mathrm{~mm}$ diameter and $5.5 \mathrm{~cm}$ in length plastic rods for the marking of the spinous processes for better side viewing; all fixed with double-sided adhesive tape (Fig. 1). On the sagittal view, they were requested to bend their elbows at $90^{\circ}$ enabling the visualization of the marker located in the anterior-superior iliac spine (ASIS). Each evaluation lasted about 45 minutes, having their parents or guardians presence at all procedures. Digital photos were transferred to the SAPO software (version 0.68), which allowed us to have the description of global postural analysis; center of gravity projection (CGP) and spine analysis in the sagittal
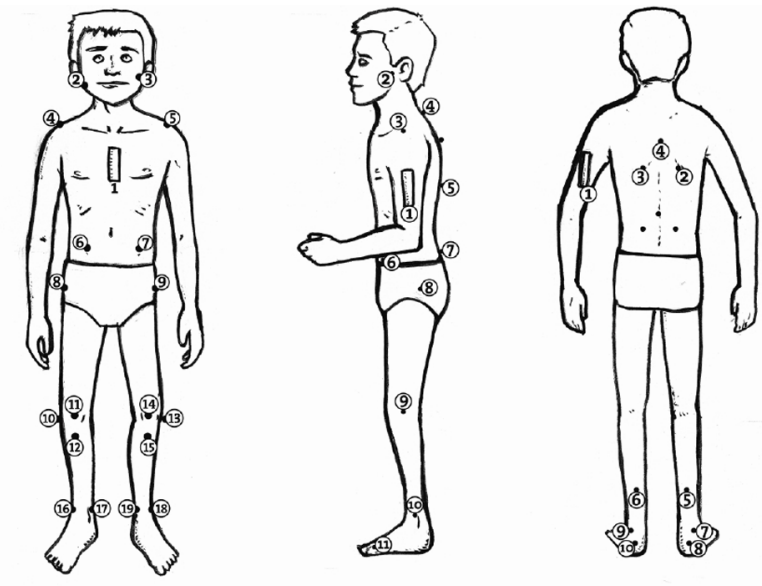

Elaborate by Matheus Brignol Nobre. Artwork: Mateus Arrigon

In all views photographed was used a tape as vertical reference for further analysis and calibration (1). Anatomical points marked in the anterior view $(2,3$ ear lobe; 4, 5 acromion; 6, 7 anterior-superior iliac spine; 8, 9 greater trochanter of femur; 10, 13 knee joint line; 11, 14 center of the patella; 12, 15 tibial tuberosity 16, 18 lateral maleoli; 17,19 medial maleoli). Left side view (2 ear lobe; 3 16, 18 lateral maleoli; 17, 19 medial maleoli). Left side view (2 ear lobe; 3 acromion; 4 seventh cervical vertebra; 5 twelfth thoracic vertebra; 6
anterior-superior iliac spine; 7 posterior-superior iliac spine; 8 greater trochanter of femur; 9 knee joint line; 10 lateral malleulus; 11 point between the second and third metatarsal heads). In the posterior view (2, 3 inferior angle of the scapula; 4 third thoracic vertebra; 5,6 point on the midline of the leg; 7, 9 Achilles tendon; 8,10 calcaneus).

Fig. 1. References used for postural analysis

plane. The software for postural assessment SAPO is a free software developed with national research funding (Research Project: "Free software for postural evaluation, with database and scientific tutorials" Nov/2003-Nov/2005, São Paulo, Brazil- Support: Brazilian Minister of Science and Technology, and Scientific and Technological Development Council) and it is freely available at http://puig.pro.br/sapo/, being a valid, reliable and reproducible instrument ${ }^{17)}$. For the postural analysis in children, the SAPO program proved to be a feasible and reliable quantitative method once there was high agreement values of all measurements performed by three different examiners (no ICC was less than 0.80$)^{18)}$.

Photograph analysis was conducted according to the following procedure: Stock photography opening at $100 \%$ zoom, image calibration, marking of points following the SAPO protocol (Table 1). For analysis in the sagittal view Perry et al. ${ }^{19)}$ recommend the use of easily palpable anatomical regions and the largest distance between the anatomical markers in order to maximize reliability. Thus, three angular measurements were obtained to describe the thoraco-lumbopelvic alignment, with the method based on the studies by Perry et al. ${ }^{19)}$, Smith; O'Sullivan; Straker ${ }^{20)}$, Smith et al. ${ }^{21)}$, and $\mathrm{Camargo}^{22)}: 1$. Trunk angle: formed between $\mathrm{C} 7$ to T12 and $\mathrm{T} 12$ to the greater trochanter (posterior angle of intersection); 2. Lumbar angle: between the line of T12 to the ASIS and ASIS to the greater trochanter (posterior angle); and 3. Sway angle: between C7 line to the greater trochanter and the greater trochanter to the lateral malleolus. The inclination to the right and left are expressed by signals, the positive value being used to express the anti-clockwise and the negative one for clockwise ${ }^{23,24)}$. Following the study, a written feedback of the results evaluation and referrals was given to 
Table 1. Measures used by the protocol of the SAPO software

\begin{tabular}{|c|c|}
\hline \multicolumn{2}{|c|}{ Anterior view } \\
\hline Head & A1- horizontal alignment of the head \\
\hline \multirow[t]{3}{*}{ Trunk } & A2- horizontal alignment of the acromia \\
\hline & A3- horizontal alignment of the ASIS \\
\hline & A4- angle between the two acromia and the two ASIs \\
\hline \multirow{6}{*}{$\begin{array}{l}\text { Lower } \\
\text { limbs }\end{array}$} & A5- frontal angle of the right lower limb \\
\hline & A6- frontal angle of the left lower limb \\
\hline & A7- difference in lower limb length: right - left sides \\
\hline & A8- horizontal alignment of the tibial tuberosities \\
\hline & A9- right $Q$ angle \\
\hline & A10- left Q angle \\
\hline \multicolumn{2}{|c|}{ Left lateral view } \\
\hline \multirow[t]{2}{*}{ Head } & A11- horizontal alignment of the head (C7) \\
\hline & A12- vertical alignment of the head (acromion) \\
\hline \multirow[t]{4}{*}{ Trunk } & A13-vertical alignment of the trunk \\
\hline & A14- hip angle (trunk and lower limb) \\
\hline & A15- vertical alignment of the body \\
\hline & A16- horizontal alignment of the pelvis \\
\hline \multicolumn{2}{|c|}{ Lower A17- knee angle } \\
\hline limbs & A18- angle of ankle \\
\hline \multicolumn{2}{|c|}{ Posterior view } \\
\hline \multicolumn{2}{|c|}{ Trunk horizontal asymmetry of the scapula in relation to $\mathrm{T}$} \\
\hline Lower & A19- leg/right hindfoot angle \\
\hline limbs & A20- leg/left hindfoot angle \\
\hline
\end{tabular}

ASIS: anterior superior iliac spines; C7: seventh cervical vertebrae; T3: thirteen thoracic vertebrae

each participant and to the responsible for the BCT.

The data were analyzed in the GraphPad Prism ${ }^{\circledR} 6$ statistical program. The distribution of normality was verified by the Shapiro-Wilk test, the values being presented in means and standard deviations or median and interquartile ranges [25-75]. The paired t-Student or Wilcoxon tests were used to compare the values obtained from the analysis of the right and left side of the body and the Mann-Whitney test to compare groups of children and adolescents. Significance was set at $5 \%$.

\section{RESULTS}

We evaluated 21 children and adolescents victims of burn injuries, at the age [mean $\pm \mathrm{SD}$ ], $10.0 \pm 2.7$ years, 16 males and five females, nine children $(7.3 \pm 1.1$ years) and 12 adolescents $(12.0 \pm 1.4 \text { years })^{25)}$ with $12.7 \pm 5.5$ months after hospital discharge. All children reported no pain at the time of evaluation and two teenagers reported mild pain associated with the burns (Table 2). The values obtained by the SAPO protocol with the anterior, lateral and posterior left views are shown in Table 3. Sagittal plane data were presented only on the left side view, since there was no statistically significant difference between the values obtained by analysis of the right and left lateral view. The analysis of the values obtained by the thoraco-lumbo-pelvic alignment is shown in Table 4.

Most children and adolescents values obtained through the SAPO protocol were different from the reference values, moreover head and trunk alignment discrepancies for head, trunk and deviation of the CGP were observed in both groups. There were no statistically significant differences between children and adolescents values in the thoraco-lumbo-pelvic alignment analysis.

\section{DISCUSSION}

The present study assessed 21 children and adolescents, classified as medium and large burns; of these, only two teenagers reported mild pain associated with the burns. The group consisting of adolescents showed higher body surface area burned, and underwent a bigger number of surgical procedures when compared to the children's group. This shows that, in some teenagers, even six months after hospital discharge, there could be pain associated with burns. One could note that in the study by Schneider et al. ${ }^{26)}$, the frequency and intensity of pain were reduced within two years after the burn, however it was still present in children victims of burns. In the outpatient setting, the presence of pain related to burning should be considered in treatment planning.

Ferreira et al. ${ }^{23)}$, used zero as parameters for the horizontal alignment of the head, acromion, pelvis (ASIS) and trunk (angle between acromion and ASIS). In this study, the measures that represent these parameters are, respectively, $\mathrm{A} 1, \mathrm{~A} 2, \mathrm{~A} 3$ and A4. When analyzing these results children and adolescents had similar values which were close to zero; except for the pelvis which were significant difference between the groups, with children presenting pelvic inclination more frequent on the left (high right pelvis), unlike adolescents who had a higher frequency of inclination to the right (higher left pelvis) and more distant values of zero. Such values are identified when facing the CGP, in which such projection in the frontal plane, showed no statistically significant difference between groups. Children had a higher frequency of weight transfer to the left and adolescents to the right, with more distant values of zero as well.

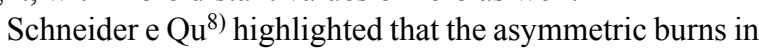
the trunk, hips and shoulder girdle can lead children to favor the affected side, that is, an inclination towards the burned area. Quiu et al. ${ }^{27)}$, also reported that wound contracture, in the trunk can lead to imbalance in the lateral-spine. In this context, most children and adolescents had their trunks affected by the burn and in accordance with Quiu et al. ${ }^{27)}$, it was possible to verify the lateral deviation in both groups, as well as the individual analysis made it possible to notice the asymmetry of the injury, however, it was not possible to notice the detection of any pattern to explain the difference in weight transfer between the groups. For the analysis of the lower limbs no significant difference was observed, as well as in the analysis of the difference of length of the lower limbs (A7), the average values were close to zero. Simultaneously, when considering the alignment of tibias (A8), both in children and adolescents value zero suggests horizontal alignment, as for the frontal angle (A7 and A8). The only reference values, defined at the SAPO report are the measures for the $\mathrm{Q}(\mathrm{A} 9$ and $\mathrm{A} 10)$ angle, whose reference value is of $15^{\circ}$, thus, the values obtained in this sample are close not only to the reference value but also to the values 
Table 2. Characteristics of children and adolescents victims of burns during the hospital stay and after discharge (at the time of evaluation)

\begin{tabular}{lcc}
\hline & $\begin{array}{c}\text { Children } \\
(\mathrm{N}=9)\end{array}$ & $\begin{array}{c}\text { Adolescents } \\
(\mathrm{N}=12)\end{array}$ \\
\hline Hospital stay & & \\
BSAB (\%), median [25 to 75\%] & $7[5.5$ to 11.2$]$ & $8.5[2.5$ to 15.0$]$ \\
Major burn, No.(\%) & $7(77.7)$ & $10(83.3)$ \\
Average burn, No.(\%) & $2(22.2)$ & $2(16.6)$ \\
Second Degree burn, No.(\%) & $5(55.5)$ & $7(58.3)$ \\
Third Degree burn, No.(\%) & $4(44.4)$ & $5(41.6)$ \\
Length of Stay (days), & $10[8.5$ to 16.0$]$ & $14.5[11.2$ to 17.7$]$ \\
median [25 to 75\%] & & \\
Home environment, No. (\%) & $9(100.0)$ & $11(91.6)$ \\
Attempted murder, No. (\%) & 0 & $1(8.3)$ \\
Facial burns and/or neck,No.(\%) & $3(33.3)$ & $9(75.0)$ \\
Anterior trunk burning, No. (\%) & $4(44.4)$ & $7(58.3)$ \\
Burn rear trunk, No. (\%) & $2(22.2)$ & $3(25.0)$ \\
Burn upper limbs, No.(\%) & $5(55.5)$ & $8(66.6)$ \\
Burn the lower limbs, No.(\%) & $5(55.5)$ & $4(33.3)$ \\
Intensive Care Unit, No.(\%) & 0 & $2(16.6)$ \\
Mechnical Ventilation, No.(\%) & 0 & $2(16.6)$ \\
Debridement, No.(\%) & $6(66.6)$ & $10(83.3)$ \\
Escharotomy, No.(\%) & 0 & $1(8.3)$ \\
Grafting, No.(\%) & $6(66.6)$ & $8(66.6)$ \\
Physical therapy, No.(\%) & $9(100.0)$ & $12(100.0)$ \\
\hline Evaluation data & & \\
Absence of pain, No. (\%) & $9(100.0)$ & $2(16.6)$ \\
Mild pain related to burning, No.(\%) & 0 & \\
Outpatient physical therapy, No.(\%) & & \\
\hline BSA & & \\
\hline body sin) & \\
\hline
\end{tabular}

BSAB: body surface area burned; No: number

found in the study carried out by Souza et al. ${ }^{24)}$, with young adults that presented values slightly higher than the ones of the reference.

In the lateral view, the vertical alignment of the head was the only value that presented a statistically meaningful difference among the groups, but there are no normality parameters for the studied age. In clinical practice, when analyzing the points that make up the A12 angle, it is possible to infer that the further tragus is in relation to the acromion the biggest the positive value of this angulation, thus, both forward head and shoulder protrusion, could change such angulation, and it is what makes difficult to interpret this meaningful difference between the groups. The angle that represents the head positioning in relation to the neutral or anterior position is the $\mathrm{C} 7$, ear lobule and the horizontal alignment of the head (A11), based on this angle the study carried out with adolescents by Ruivo, Pezarat-Correia and Carita ${ }^{28)}$, established that the values smaller than $50^{\circ}$ indicate forward head posture and therefore, it may be said that in the current study, both children and adolescents presented forward head posture. When analyzing the projection of the CG in the sagittal plane, it can be observed that children and adolescents have body weight forwardly distributed. For other angular values no references in the literature was found regarding the studied age group. In posterior view, Ferreira et al. ${ }^{23)}$ considered zero value in the scapular alignment in relation to T3, having found an average value of 0.5 that differs from the one found in our study, in which, the results suggest scapular asymmetry that occurred in almost $77 \%$ of children and $83 \%$ of adolescents from this study.

SAPO protocol has restricted information about the thoraco-lumbo-pelvic alignment, therefore this study used the methodology described in the study by Perry et al. ${ }^{19)}$, which when analyzing the reliability of photographic analysis of the standing posture in the sagittal plane in adolescents, found excellent intra-examiner (ICC $=0.99$ for the three angles, with standard error of 0.2 for the angle of body inclination and 0.4 for the angles of the trunk and lumbar), thus suggesting a good reproducibility test. As for the inter-rater reliability analysis, these same measures varied from poor to reasonable suggesting that factors such as the placement of markers, setting parameters, body positioning, and biological variability, may have led to lower reliability. For the interpretation of the measures obtained from the thoracic lumbar-pelvic alignment in children the study used was the one from Camargo ${ }^{22)}$, which presented as values: the average of inclination angle of $163.4 \pm 4.8$; trunk angle of $203.1 \pm 7.9$ and lumbar angle of $87.5 \pm 8.8$. In the present 
Table 3. Values obtained through the SAPO protocol and comparasion between children and adolescents

\begin{tabular}{|c|c|c|c|c|}
\hline & Measure & $\begin{array}{l}\text { Children } \\
(\mathrm{N}=9)\end{array}$ & $\begin{array}{l}\text { Adolescents } \\
(\mathrm{N}=12)\end{array}$ & $\begin{array}{c}\text { Reference } \\
\text { (SAPO protocol) }\end{array}$ \\
\hline \multicolumn{5}{|l|}{ Anterior } \\
\hline Head & $\mathrm{A} 1$ & $1.3[-3.4$ to 3.4$]$ & $1.5[0.3$ to 6.6$]$ & 0 \\
\hline \multirow[t]{3}{*}{ Trunk } & $\mathrm{A} 2$ & $-0.9[-2.1$ to 1.1$]$ & $1.7[0.0$ to 3.2$]$ & 0 \\
\hline & $\mathrm{A} 3$ & $-1.0[-2.4$ to 0.0$]$ & $1.0[0.0$ to 3.7$] *$ & 0 \\
\hline & A4 & $0.0[-1.8$ to 3.7$]$ & $0.1[-1.2$ to 2.5$]$ & 0 \\
\hline Lower & A5 & $-0.4[-1.6$ to 1.1$]$ & $-0.6[-3.2$ to 0.0$]$ & NA \\
\hline \multirow[t]{5}{*}{ Limbs } & A6 & $-1.0[-1.8$ to 0.2$]$ & $0.0[-1.5$ to 1.7$]$ & NA \\
\hline & A7 & $0.6[-0.7$ to 1.3$]$ & $-0.6[-1.3$ to 0.4$]$ & NA \\
\hline & A8 & $0.0[-1.6$ to 0.0$]$ & $0.0[-1.4$ to 1.5$]$ & 0 \\
\hline & A9 & 10.5 [7.5 to 19.9$]$ & $13.8[10.9$ to 15.8$]$ & 15 \\
\hline & A10 & 20.2 [19.4 to 24.3$]$ & $16.6[8.4$ to 24.8$]$ & 15 \\
\hline \multicolumn{5}{|l|}{ Left lateral view } \\
\hline \multirow[t]{2}{*}{ Head } & A11 & 49.9 [42.8 to 54.3$]$ & $48.5[43.4$ to 54.0$]$ & NA \\
\hline & $\mathrm{A} 12$ & $12.3[9.2$ to 18.9$]$ & $21.4[14.2 \text { to } 26.4]^{*}$ & 0 \\
\hline \multirow[t]{4}{*}{ Trunk } & A13 & $-6.4[-6.9$ to 1.7$]$ & $-5.7[-8.9$ to -0.1$]$ & NA \\
\hline & A14 & $-12.0[-15.8$ to -4.8$]$ & $-10.9[-16.6$ to -5.7$]$ & NA \\
\hline & A15 & $0.2[-0.7$ to 1.2$]$ & $1.0[-0.5$ to 3.8$]$ & NA \\
\hline & A16 & $-16.4[-18.6 ;-8.2]$ & $-15.3[-19.7$ to -9.3$]$ & NA \\
\hline Lower & A17 & $-5.3[-9.5$ to -1.5$]$ & $-1.4[-5.2$ to 5.2$]$ & NA \\
\hline Limbs & A18 & 90.0 [87.2 to 93.2$]$ & 86.4 [80.1 to 89.5$]$ & NA \\
\hline \multicolumn{5}{|l|}{ Posterior } \\
\hline Trunk & Assim & $-6.9[-18.5$ to 7.4$]$ & $2.0[-15.6$ to 10.8$]$ & 0 \\
\hline Lower & A19 & $13.7[4.3$ to 15.8$]$ & $6.0[2.1$ to 9.0$]$ & NA \\
\hline Limbs & A 20 & 11.1 [4.3 to 12.8$]$ & $6.2[2.4$ to 12.2$]$ & NA \\
\hline CGP- frontal plane (\%) & & $-5.8[-15.3$ to 4.8$]$ & $8.6[-8.7$ to 20.0$]$ & 0 \\
\hline CGP- sagittal plane (\%) & & 22.1 [16.2 to 27.3$]$ & 36.1 [19.0 to 39.8$]$ & 0 \\
\hline
\end{tabular}

$* \mathrm{p}<0.05$; median [25 to $75 \%$ ]; Positive values indicate tilt to the right and negative to the left;

Assim: horizontal asymmetry of the scapula in relation to T3; CGP: Center of Gravity Projection; NA: Not available

Table 4. Values obtained by analyzing the thoraco-lumbo-pelvic region and comparasion between children and adolescents

\begin{tabular}{lcc}
\hline Measures & $\begin{array}{c}\text { Children } \\
(\mathrm{N}=9)\end{array}$ & $\begin{array}{c}\text { Adolescents } \\
(\mathrm{N}=12)\end{array}$ \\
\hline Trunk Angle & $207.1[203.7$ to 211.8$]$ & $205.8[203.2$ to 211.0$]$ \\
Lumbar Angle & $86.6[82.4$ to 89.1$]$ & $83.5[82.1$ to 85.4$]$ \\
Sway Angle & $166.0[164.0$ to 167.6$]$ & $166.5[165.2$ to 167.1$]$ \\
\hline$* \mathrm{p}>0.05 ;$ median $[25$ to $75 \%]$ &
\end{tabular}

study it was possible to observe that, children presented a bigger inclination angle (tendency to anterior inclination of the trunk) with a bigger trunk angle (thoracic kyphosis); and a smaller lumbar angle (increased lumbar lordosis). As for the adolescents the study by Smith et al. ${ }^{21)}$, was used as reference, being considered: neutral inclination angle of $166.5 \pm 4.2$; neutral trunk angle of $201.7 \pm 6.1$ and neutral lumbar angle of $86.5 \pm 6.9$. In the present study, it was found that adolescents had inclination angle within the considered neutral position; however, with increased thoracic kyphosis and increased lumbar lordosis. It is important to highlight the fact that about $33 \%$ of children and $75 \%$ of adolescents had burns involving face and/or cervical; and $44 \%$ of children and $58 \%$ of adolescents had the anterior trunk affected. Schneider e $\mathrm{Qu}^{8}$, point out that burns in childhood, in anterior cervical and thoracic region can lead to anterior shoulder and kyphotic posture, along with a protection posture. Furthermore, wound contracture in burned patients, in the trunk region, may biomechanically induce to increased lordosis, depending on the affected region ${ }^{27)}$.

One limitation of the study is the reduced number of samples, due to the difficulty of recruiting subjects, as the 
BTC is a reference center for treating victims of burns, most of the individuals did not attend the outpatient return due to transportation problems. We point out the difficulty in comparing the results with those found in the literature by using different methodologies and the lack of normal reference values for the age range considered.

This study highlights some considerations for further research, such as psychological aspects, which was not investigated. Prykora et al. ${ }^{29)}$ argues that extensive burns are considered great physical and psychological traumatic events in the life of children. Some studies have been conducted to verify the reciprocal association between emotional state and posture ${ }^{30}$. As for the clinical implications, the study demonstrates the need for more careful attention to the postural aspect in pediatric burn victims after hospital discharge, since it was observed as in children and adolescents with multiple affected body regions as in those with injuries in only one segment, postural deviation in both planes - the frontal plane, with greater weight transfer on one side and/ or scapular asymmetry; as the sagittal plane, with changes in segments of the head and/or thoracic region and/or lumbar region. These results indicate that, in this specific population, there is an increased risk for the development of pain and even future scoliosis, requiring postural monitoring until the end of the growth period.

\section{ACNOWLEDGEMENTS}

The authors would like to express their appreciation to all children and adolescents who participated in this study and we thank Matheus Brignol Nobre and Mateus Arrigoni for the drawing and artwork, respectively.

\section{REFERENCES}

1) World Health Organization (WHO): A WHO plan for burn prevention and care. Geneva, Switzerland, 2008. http://whqlibdoc.who.int/publications/2008/9789241596299 eng.pdf (Accessed Jun. 22, 2014).

2) Stubbs TK, James LE, Daugherty MB, et al.: Psychosocial impact of childhood face burns: a multicenter, prospective, longitudinal study of $390 \mathrm{chil}-$ dren and adolescents. Burns, 2011, 37: 387-394. [Medline] [CrossRef]

3) Davoodi P, Fernandez JM, O SJ: Postburn sequelae in the pediatric patient: clinical presentations and treatment options. J Craniofac Surg, 2008, 19: 1047-1052. [Medline] [CrossRef]

4) Forjuoh SN: Burns in low- and middle-income countries: a review of available literature on descriptive epidemiology, risk factors, treatment, and prevention. Burns, 2006, 32: 529-537. [Medline] [CrossRef]

5) Lloyd EC, Rodgers BC, Michener M, et al.: Outpatient burns: prevention and care. Am Fam Physician, 2012, 85: 25-32. [Medline]

6) Berman B, Viera MH, Amini S, et al.: Prevention and management of hypertrophic scars and keloids after burns in children. J Craniofac Surg, 2008, 19: 989-1006. [Medline] [CrossRef]

7) Wiechman SA: Psychosocial recovery, pain, and itch after burn injuries Phys Med Rehabil Clin N Am, 2011, 22: 327-345, vii. [Medline] [CrossRef]

8) Schneider JC, Qu HD: Neurologic and musculoskeletal complications of burn injuries. Phys Med Rehabil Clin N Am, 2011, 22: 261-275, vi. [Med- line] [CrossRef]

9) Heath K, Timbrell V, Calvert P, et al.: Outcome measurement tools currently used to assess pediatric burn patients: an occupational therapy and physiotherapy perspective. J Burn Care Res, 2011, 32: 600-607. [Medline] [CrossRef]

10) Ali ZM, El-Refay BH, Ali RR: Aerobic exercise training in modulation of aerobic physical fitness and balance of burned patients. J Phys Ther Sci, 2015, 27: 585-589. [Medline] [CrossRef]

11) Serghiou M, Cowan A, Whitehead C: Rehabilitation after a burn injury. Clin Plast Surg, 2009, 36: 675-686. [Medline] [CrossRef]

12) Shaheen AA, Basuodan RM: Quantitation assessment of head posture of young adults based on lateral view photographs. J Phys Ther Sci, 2012, 24: 391-394. [CrossRef]

13) Nam SH, Son SM, Kwon JW, et al.: The intra- and inter-rater reliabilities of the forward head posture assessment of normal healthy subjects. J Phys Ther Sci, 2013, 25: 737-739. [Medline] [CrossRef]

14) Claro MT: Escala de faces para avaliação da dor em crianças: etapa preliminar. Dissertação (Mestrado)-Escola de Enfermagem de Ribeirão Preto, Universidade de São Paulo. São Paulo: Ribeirão Preto, 1993. p 50.

15) Ferreira EA: Postura e controle postural: desenvolvimento e aplicação de método quantitativo de avaliação postural. [Tese]. São Paulo: Faculdade de Medicina, Universidade de São Paulo, 2005. p.114.

16) Dolphens M, Cagnie B, Coorevits $P$, et al.: Classification system of the normal variation in sagittal standing plane alignment: a study among young adolescent boys. Spine, 2013, 38: E1003-E1012. [Medline] [CrossRef]

17) Ferreira EA, Duarte M, Maldonado EP, et al.: Postural assessment software (PAS/SAPO): validation and reliabiliy. Clinics (Sao Paulo), 2010, 65: 675-681. [Medline] [CrossRef]

18) Santos MM, Silva MP, Sanada LS, et al.: Photogrammetric postural analysis on healthy seven to ten-year-old children: interrater reliability. Rev Bras Fisioter, 2009, 13: 350-355. [CrossRef]

19) Perry M, Smith A, Straker L, et al.: Reliability of sagittal photographic spinal posture assessment in adolescents. Adv Physiother, 2008, 10: 66-75. [CrossRef]

20) Smith A, O'Sullivan P, Straker L: Classification of sagittal thoraco-lumbopelvic alignment of the adolescent spine in standing and its relationship to low back pain. Spine, 2008, 33: 2101-2107. [Medline] [CrossRef]

21) Smith AJ, O'Sullivan PB, Beales DJ, et al.: Trajectories of childhood body mass index are associated with adolescent sagittal standing posture. Int $\mathbf{J}$ Pediatr Obes, 2011, 6: e97-e106. [Medline] [CrossRef]

22) Camargo MZ: Postura e obesidade infantil: análise do alinhamento no plano sagital em pré-escolares. [tese] Universidade Estadual de Londrina, Londrina. 2013. 60f.

23) Ferreira EA, Duarte M, Maldonado EP, et al.: Quantitative assessment of postural alignment in young adults based on photographs of anterior, posterior, and lateral views. J Manipulative Physiol Ther, 2011, 34: 371-380. [Medline] [CrossRef]

24) Souza JA, Pasinato F, Basso D, et al.: Biophotogrammetry: reliability of measurements obtained with a posture assessment software (SAPO). Rev Bras Cineantropom Desempenho Hum, 2011, 13: 299-305.

25) WHO, World Health Organization: Adolescent health. http://www.who.int/ topics/adolescent_health/en/(Accessed Jun. 22, 2014).

26) Schneider JC, Nadler DL, Herndon DN, et al.: Pruritus in pediatric burn survivors: defining the clinical course. J Burn Care Res, 2015, 36: 151-158. [Medline] [CrossRef]

27) Qiu Y, Wang SF, Wang B, et al.: Adolescent scar contracture scoliosis caused by back scalding during the infantile period. Eur Spine J, 2007, 16: 1557-1562. [Medline] [CrossRef]

28) Ruivo RM, Pezarat-Correia P, Carita AI: Cervical and shoulder postural assessment of adolescents between 15 and 17 years old and association with upper quadrant pain. Braz J Phys Ther, 2014, 18: 364-371. [Medline] [CrossRef]

29) Przkora R, Barrow RE, Jeschke MG, et al.: Body composition changes with time in pediatric burn patients. J Trauma, 2006, 60: 968-971, discussion 971. [Medline] [CrossRef]

30) Oosterwijk S, Rotteveel M, Fischer AH, et al.: Embodied emotion concepts: how generating words about pride and disappointment influences posture. Eur J Soc Psychol, 2009, 39: 457-466. [CrossRef] 